\title{
¿Es necesario realizar un estudio de etapificación en todos los pacientes con cáncer de próstata? Experiencia de un centro clínico nacional
}

\author{
NAGEL MARTÍNEZ M. ${ }^{1}$, CARLOS CALVO $^{a}$, ÁLVARO IBARRA², \\ CHRISTIAN RAMOS ${ }^{3}$, NORMAN ZAMBRANO ${ }^{3}$
}

\section{Usefulness of imaging studies in prostate cancer. Analysis of 241 patients}

Background: The role of staging studies in patients with prostate cancer (PCa) is a topic of discussion. Aim: To evaluate the usefulness of imaging studies in patients with prostate cancer. Material and Methods: We reviewed the pathology service records to identify patients with prostate cancer diagnosed between 2003 and 2013. We reviewed the electronic medical records of those patients identified as having a prostate cancer. Patients were grouped according D'amico's classification of cancer dissemination risk. We analized the frequency of imaging studies requested and their efficacy to detect metastases in each risk group. Results: We identified 241 patients with a mean age of 67 years. Fifty two percent of patients were classified as low-risk, 32\% as intermediate-risk and $16 \%$ as high risk. At least one imaging study was requested to $64 \%$ of patients (49, 78 and $87 \%$ of patients with low, intermediate and high risk respectively). Among the 155 patients in whom an imaging study was requested, no metastases were found in the low risk group. On the other hand, dissemination was found in $7 \%$ of the intermediate-risk group and $62 \%$ of the high-risk group. Conclusions: Half of patients with prostate cancer were classified as low risk. In half of this group of low risk patients, staging studies were requested and the probability of detecting metastases was low or nil. The odds of detecting metastases increased in higher risk groups.

(Rev Med Chile 2017; 145: 579-584) Risk.

Key words: Neoplasm Metastasis; Neoplasm Staging; Prostatic Neoplasms;

$\mathrm{E}$ 1 cáncer de próstata $(\mathrm{CP})$ es el tumor de mayor frecuencia y el segundo más letal en la población masculina de Estados Unidos de Norteamérica ${ }^{1}$. A nivel mundial ha adquirido mayor importancia por el incremento progresivo de su incidencia y mortalidad ${ }^{2}$. En el caso de Chile, el escenario epidemiológico actual se conforma por una prevalencia que alcanza 9,2 casos por 1.000 individuos y una tasa de mortalidad que durante el 2013 fue de 23,4/100.000 habitantes ${ }^{3}$.

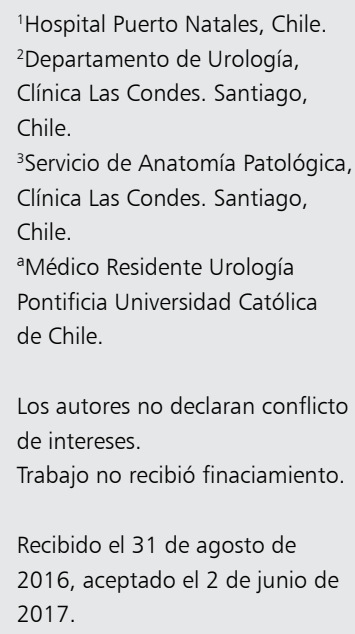

Los autores no declaran conflicto de intereses.

Trabajo no recibió finaciamiento.

Recibido el 31 de agosto de 2016, aceptado el 2 de junio de 2017.

\section{Correspondencia a:}

Dr. Nagel Martínez Molina Hospital Puerto Natales, Chile. nagomartinez@gmail.com
Esto evidencia lo trascendente que es desarrollar adecuados métodos de tamizaje, pruebas diagnósticas y estudios de diseminación.

Algunos de estos métodos, como el tacto rectal y el antígeno prostático específico (APE), permiten sospechar y realizar un diagnóstico precoz de neoplasia prostática y, por tanto, detectar un cáncer generalmente confinado a la próstata ${ }^{1}$. Esto implica que al momento del diagnóstico, la mayoría de los pacientes tengan una enferme- 
dad poco avanzada y sean considerados para un tratamiento con intención curativa, como son la prostatectomía radical y la radioterapia ${ }^{4}$.

Por lo tanto, la detección de lesiones secundarias en pacientes con CP es crucial para decidir la conducta terapéutica, ya que en caso de existir diseminación, generalmente se desestiman las terapias con intención curativa ${ }^{5-7}$.

Existen algunos factores, tanto clínicos como de laboratorio, que han sido estudiados como predictores de diseminación en CP. Uno de ellos es el antígeno prostático específico (APE), marcador proteico cuyo incremento se correlaciona tanto con el crecimiento de masa tumoral como con la presencia de lesiones secundarias. Ejemplo de esto es una muestra de 683 pacientes, en que Gleave y cols., mostraron, retrospectivamente, que individuos con APE menor a $10 \mathrm{ng} / \mathrm{ml}$ no tenían lesiones secundarias en el cintigrama óseo. En cambio, $40 \%$ de los pacientes con APE mayor a $50 \mathrm{ng} / \mathrm{ml}$ tenía un examen positivo para diseminación ${ }^{8}$. Así mismo, en los casos de un APE mayor a $100 \mathrm{ng} /$ $\mathrm{ml}$, el valor predictivo positivo del cintigrama fue de $100 \%$ para lesiones óseas.

Otro factor es el puntaje de Gleason, sistema que mide el grado de diferenciación celular en la muestra histológica de la próstata. En una investigación en 932 pacientes, en los que se realizó una prostatectomía más disección ganglionar, no se encontró compromiso metastásico en ninguna de las muestras con Gleason $\leq 5^{9}$. Así mismo, otros estudios en pacientes con puntaje de Gleason $\geq 8$, evidencian un mayor número de casos con diseminación secundaria ${ }^{10}$, lo que revela su rol predictor de riesgo de metástasis.

El estadío clínico, definido por consenso según la clasificación $\mathrm{TNM}^{11}$, también es utilizado como factor predictor de riesgo de lesiones secundarias. Si se evalúa este criterio como variable aislada, se observa una tendencia, con $80 \%$ de precisión, a encontrar más casos de diseminación en estadíos avanzados de la enfermedad (T3-T4), y prácticamente ningún caso en estadíos $\mathrm{T} 1 \mathrm{a}-\mathrm{c}^{10}$. Se han desarrollado modelos de predicción multifactorial de enfermedad metastásica. Un ejemplo de estas son las Tablas de Partin ${ }^{12}$, que muestran que un paciente con Gleason 4+3, un APE entre 4, 1 y $6 \mathrm{ng} /$ $\mathrm{ml}$ y con estadío clínico T1c, tiene 3\% de riesgo de metástasis ganglionar. En cambio, este mismo paciente, con un estadío clínico T2b/c tiene un riesgo de $11 \%$.
La evidencia expuesta en la literatura y las recomendaciones de las agrupaciones internacionales, apoyan un análisis que involucre una combinación de factores. Se prefiere combinar la concentración sérica de APE, puntuación de Gleason en la biopsia de próstata y estadío T clínico como factores predictores del riesgo de lesiones secundarias y estadío final de la enfermedad. Este modelo de análisis resulta superior al compararlo con los parámetros mencionados utilizados en forma independiente, lográndose mayor precisión al estimar el riesgo de diseminación del paciente al momento del diagnóstico ${ }^{5-7,10,13-16}$.

A pesar de que la evidencia apoya los factores mencionados como predictores de lesiones secundarias, no existe una conducta estandarizada que establezca cuáles son los pacientes que realmente necesitan un estudio de diseminación por imágenes al momento del diagnóstico ${ }^{10,14}$. Ejemplo de esto son las múltiples recomendaciones emitidas por las agrupaciones internacionales (Tabla 1).

A la luz de lo expuesto, podemos afirmar que existe un grupo de pacientes con muy bajo riesgo de metástasis, en el que los estudios de diseminación preoperatoria por imágenes no serían necesarios en forma rutinaria ${ }^{10,14,17-20}$.

En nuestro centro, solicitamos estudio con TAC de abdomen y pelvis, así como cintigrama óseo a la mayor parte de los pacientes candidatos a tratamiento curativo. Pensamos que esta conducta no es concordante con las guías actuales, ya que lleva a un gasto excesivo al paciente, así como al riesgo asociado a dichos estudios (exposición al medio de contraste y radiación).

El objetivo de nuestro trabajo es determinar la utilidad clínica de los estudios de etapificación imagenológica en pacientes con cáncer de próstata. Nos parece interesante entregar un enfoque orientado en la conducta del estudio de diseminación neoplásica, considerando los beneficios o riesgos para el paciente. Además no existen publicaciones locales o nacionales con datos acerca de la cantidad y el rendimiento de los estudios de etapificación realizados en pacientes con cáncer de próstata. Por lo mismo, queremos poner de manifiesto la realidad en nuestro centro, con la intención de que se refleje, en lo posible, la realidad nacional. De este modo, se podría orientar a los urólogos nacionales, en la toma de decisiones respecto al estudio de etapificación de pacientes con diagnóstico reciente de cáncer de próstata. 
Tabla 1. Recomendaciones de estudio imagenológico para etapificación en cáncer de próstata

\begin{tabular}{|c|c|}
\hline Asociación Europea de Urología ${ }^{5}$ & Cintigrama óseo y TAC o RNM: APE $\geq 10$ o Gleason $\geq 7$ o cT2b \\
\hline Asociación Americana de Urología ${ }^{6}$ & $\begin{array}{l}\text { TAC o RNM: APE } \geq 25 \mathrm{ng} / \mathrm{ml} \\
\text { Cintigrama óseo: cT3 o APE } \geq 20 \mathrm{ng} / \mathrm{ml}\end{array}$ \\
\hline $\begin{array}{l}\text { National Comprehensive } \\
\text { Cancer Network }\end{array}$ & $\begin{array}{l}\text { TAC o RNM: cT3, cT4, cT1-cT2 con nomograma de predicción ganglionar de }>10 \% \\
\text { Cintigrama óseo: T1 y APE } \geq 20 \mathrm{ng} / \mathrm{ml} \text {, T2 y APE } \geq 10 \mathrm{ng} / \mathrm{ml} \text {, Gleason } \geq 8 \text {, cT3, } \\
\text { cT4, sintomáticos }\end{array}$ \\
\hline
\end{tabular}

TAC: Tomografía axial computarizada. RNM: Resonancia nuclear magnética. APE: Antígeno prostático específico.

\section{Materiales y Métodos}

Se realizó un estudio observacional, retrospectivo y analítico. Previa autorización de nuestro Comité de ética, se incluyeron pacientes mayores de 18 años, con diagnóstico de cáncer de prósta$\mathrm{ta}$, que figuraban en los registros del Servicio de Anatomía Patológica de Clínica Las Condes, entre los años 2003 y 2013. Se excluyeron los pacientes que habían recibido tratamientos previos por $\mathrm{CP}$, los pacientes con diagnóstico de recurrencia de cáncer de próstata cuyo diagnóstico inicial no fue en nuestra institución y los pacientes cuya ficha clínica fue inaccesible. Se construyó una base de datos con la información contenida en los informes de biopsia, en la ficha clínica electrónica y de papel, y en los informes de imágenes y laboratorio.

Fueron recolectadas las siguientes variables: edad, comorbilidades, niveles de APE previo al diagnóstico, detalles de biopsia prostática diagnóstica (Gleason, multifocalidad, porcentaje de biopsia alterada, compromiso linfovascular/perineural) y estudio por imágenes complementario. En estos últimos, se evaluó la presencia de metástasis única o múltiple en el cintigrama óseo y presencia de ganglios patológicos, metástasis, invasión loco-regional o compromiso óseo en el TAC de abdomen y pelvis, $\mathrm{PET}-\mathrm{CT}$ o RMN, entre otras.

Una vez obtenida la información, se clasificaron los pacientes según el riesgo de diseminación, de acuerdo a los criterios de D'Amico ${ }^{16}$ (Tabla 2).

Finalmente, se analizó la cantidad de estudios realizados en cada grupo de riesgo, y se compararon las tasas de diseminación en dichos grupos. Los datos fueron analizados a través de estadística descriptiva y al comparar variables categóricas se usó la prueba $\chi^{2}$, considerándose estadísticamente significativo un valor $\mathrm{p}<0,05$. Se utilizó el programa estadístico SPSS edición 17.

\section{Resultados}

Luego de aplicar los criterios de exclusión previamente mencionados, se pudo acceder a un total de 241 pacientes como grupo de análisis.

El promedio de edad, el antígeno prostático específico y el puntaje de Gleason, junto a la disitribución según criterios de D’Amico se muestra en la Tabla 3. Al evaluar la realización de estudios de diseminación al momento del diagnóstico, 155 pacientes $(64,3 \%)$ fueron sometidos al menos a un estudio (tomografía axial computarizada [TAC], cintigrama óseo, resonancia nuclear magnética [RNM] o PET-scan [PET-CT]). De acuerdo al grupo de riesgo, los estudios de diseminación se realizaron en $48,8 \%$ de los de bajo riesgo y en

Tabla 2. Criterios de D'Amico

\begin{tabular}{|llll|}
\hline Riesgo & TNM & APE $\mathbf{~ n g} / \mathbf{m l}$ & Gleason \\
Bajo & T1c- T2a & $\leq 10$ & $\leq 6$ \\
Intermedio & T2b & $>10$ a 20 & Gleason 7 \\
Alto & T2c & $>20$ & Gleason $\geq 8$ \\
\hline
\end{tabular}

APE: Antígeno prostático específico.

Tabla 3. Características de la muestra

\begin{tabular}{|c|c|c|}
\hline \multicolumn{2}{|l|}{$\mathrm{n}$ total } & 241 \\
\hline Edad & promedio en años & 66,7 \\
\hline Gleason score & mediana (rango) & $6(6-10)$ \\
\hline APE pre-diagnós & $\begin{array}{l}\text { Śstico } \\
\text { mediana (rango) }\end{array}$ & $6,67(2,4-89,27) \mathrm{ng} / \mathrm{dl}$ \\
\hline $\begin{array}{l}\text { Riesgo según D' } \\
\text { - Bajo } \\
\text { - Intermedio } \\
\text { - Alto }\end{array}$ & 'Amico & $\begin{array}{r}125(51,8) \\
77(31,9) \\
39(16,2)\end{array}$ \\
\hline
\end{tabular}

APE: Antígeno prostático específico. 

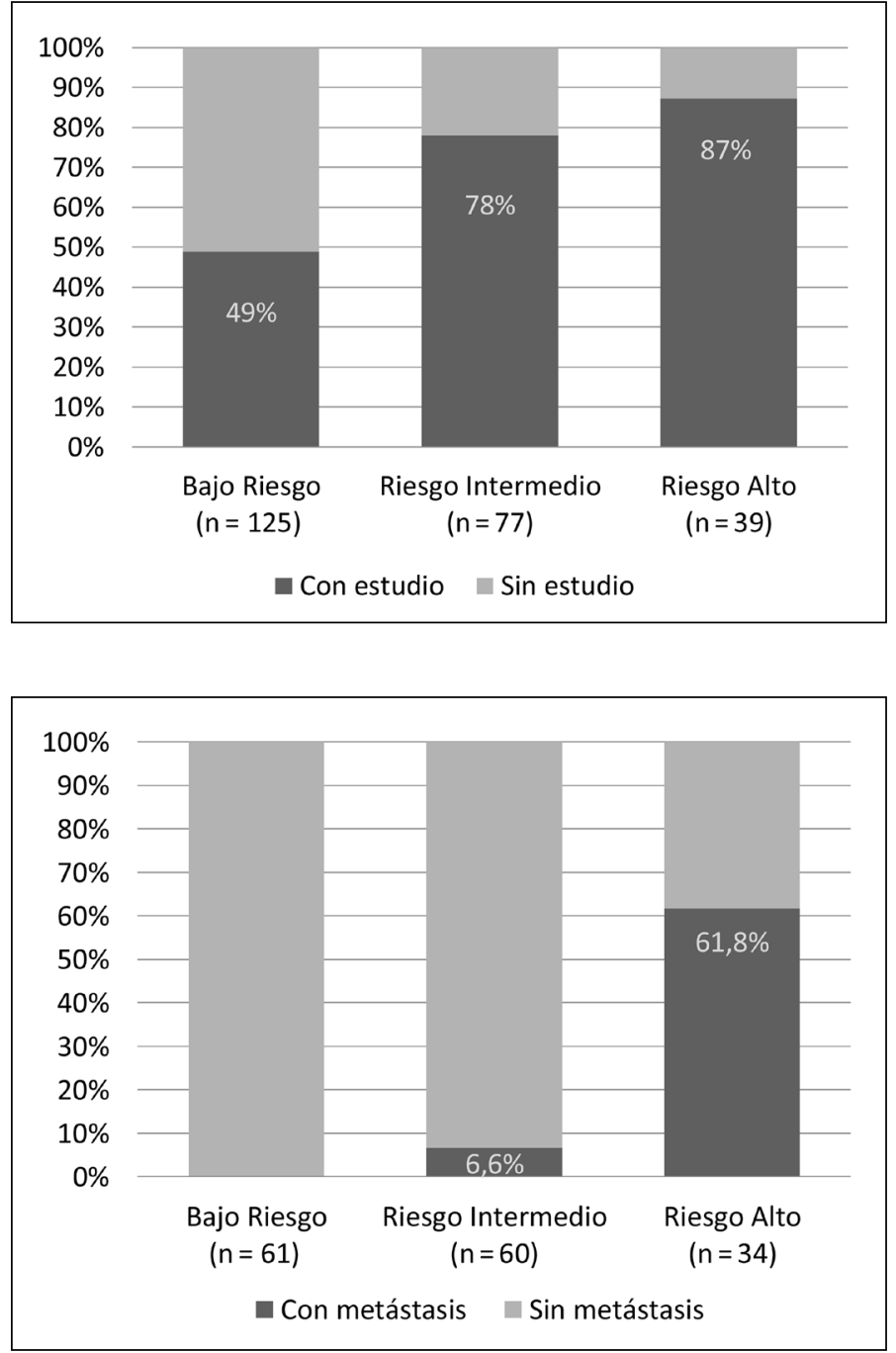

Figura 1. Proporción de pacientes con estudio de etapificación según riesgo de D’Amico.
Figura 2. Proporción de pacientes con metástasis según riesgo de D’Amico.
$77,9 \%$ y $87,2 \%$, en los de riesgo intermedio y alto, respectivamente (Figura 1). Del total de pacientes sometidos a estudio de diseminación $(\mathrm{n}=155)$, se encontró evidencias de metástasis en 6,6\% y $61,8 \%$ para los grupos de riesgo intermedio y alto, respectivamente (Figura 2). En el caso del grupo de bajo riesgo, no se detectaron pacientes con diseminación. Al observar los datos por tipo de estudio imagenológico realizado, se evidencia que el más frecuente fue TAC con 131 estudios, seguido por Cintigrama óseo, PET-CT y RNM con un total de 92, 14 y 4 estudios realizados, respectivamente. El hallazgo de metástasis en los pacientes con cáncer de próstata estuvo directamente relacionado con el grupo de riesgo (test de $\chi^{2}$, con $\mathrm{p}<0,05$ ).

\section{Discusión}

Como en toda enfermedad neoplásica, la implementación de una terapia con carácter curativo implica una correcta evaluación previa del paciente. Determinar la invasión local de la enfermedad, la presencia de enfermedad a distancia, y la relación riesgo beneficio de las terapias son elementos determinantes para tomar una decisión terapéutica adecuada.

El cáncer de próstata no es una excepción, por lo que se requiere determinar la extensión de la enfermedad, para así enfocarse a una terapia apropiada. El desafío está en identificar a los pacientes que se beneficiarían de un estudio acabado 
buscando metástasis, y separarlos del grupo de pacientes que tienen muy bajo riesgo de presentar enfermedad avanzada. De esta manera, en este artículo queremos dar énfasis en analizar la utilidad clínica de realizar estudios imagenológicos, ya que un punto importante es evitar los riesgos y disminuir los costos asociados de dichos estudios.

Dentro de nuestro universo de pacientes los casos perdidos fueron debido a la imposibilidad de recolectar todos los datos necesarios para el análisis, ya que en general, estos casos fueron diagnosticados en los primeros años de nuestro período de estudio. Además, un grupo no despreciable de pacientes con diagnóstico inicial en nuestro centro, migró posteriormente a otros establecimientos para su estudio, tratamiento y seguimientos, lo que obligó a excluirlos de nuestra población de estudio.

En lo que respecta al proceso diagnóstico del cáncer de próstata en nuestro centro, es necesario comentar que las biopsias transrectales son realizadas por distintos miembros del Servicio de Urología de Clínica Las Condes. Asimismo ocurre en el servicio de Anatomía Patológica, donde la interpretación histopatológica es realizada por tres patólogos diferentes.

$\mathrm{Al}$ analizar la distribución de los pacientes según el riesgo de D’Amico, podemos observar una predominancia de pacientes en el grupo de bajo riesgo $(51,8 \%)$. Esto podría ser explicado, en parte, por el tamizaje con APE, que ha ganado popularidad en nuestro país, sobre todo en el sector privado. Este marcador logra pesquisar los casos en estadíos más precoces, por ende, de menor riesgo y susceptibles a tratamiento curativo ${ }^{21}$. Las edades en los tres grupos son similares, lo que nos permite compararlos con mayor validez.

En cuanto a los estudio de imágenes realizados, destaca una alta tasa de realización en los grupos de riesgo intermedio y alto, con alrededor de $80 \%$ de los casos estudiados. Por otra parte, a pesar de las recomendaciones internacionales mencionadas previamente, prácticamente a la mitad de los pacientes de bajo riesgo se les realizó, al menos, un estudio de imagen. Esto refleja que la conducta entre los profesionales de nuestro grupo no es uniforme.

Los resultados de los estudios de diseminación realizados concuerdan con la literatura. Se evidenció diseminación en $6,6 \%$ y $61,8 \%$, en los grupos intermedio y alto riesgo respectivamente.
$\mathrm{Al}$ someter estas variables al test de $\chi^{2}$, muestra que el porcentaje de diseminación tiene una relación estadísticamente significativa con el riesgo según D'Amico $(\mathrm{p}<0,05)$.

Dicha observación apoya la recomendación de no realizar estudios de diseminación en pacientes de riesgo bajo. Se están realizando exámenes de alto costo económico, y con exposición a radiación ionizante a pacientes que obtienen un beneficio marginal de estos estudios. De acuerdo a la evidencia presentada, los pacientes de bajo riesgo no tienen lesión secundaria al momento del diagnóstico ${ }^{8-10}$. Esto significa que un alto volumen de pacientes se somete a exámenes radiológicos, sin una clara justificación y sin un beneficio objetivo claro.

Nuestro reporte concuerda con Lavery y cols., quien en 2011 intentó cuantificar los exámenes de imágenes (Cintigrama, TAC o RNM) tomados en pacientes que no tenían indicación según las guías internacionales. En aquella investigación definieron como grupo de bajo riesgo a los pacientes con $\mathrm{APE} \leq 10$ y Gleason $\leq 6$, y observaron que en este grupo, $48 \%$ fue sometido, al menos, a un examen de imagen y $30 \%$ fue sometido a dos exámenes innecesarios ${ }^{18}$.

Sólo para mencionar, al observar los resultados de cada grupo de riesgo se obtiene que en el de alto riesgo, PET-CT y TAC detectan mejor la diseminación que el resto de los estudios. Pero cabe indicar que sería necesario aumentar la cantidad total disponible de estos estudios en próximos trabajos para conclusiones más detalladas.

Es necesario comentar que tenemos sólo un caso en que el Cintigrama óseo resultó negativo para diseminación y el PET-CT positivo en un paciente previamente clasificado como de alto riesgo.

Para concluir, la mitad de los pacientes diagnosticados de cáncer de próstata en nuestro centro son de bajo riesgo según D'Amico. Y a pesar de las recomendaciones internacionales, se realiza algún estudio de etapificación a casi la mitad de los pacientes de este grupo. La probabilidad de detectar lesiones secundarias se correlaciona de forma significativa con el grupo de riesgo, y destaca en el grupo de bajo riesgo que la probabilidad de detectar enfermedad a distancia es bajísima. Por lo tanto, cuando nos enfocamos en la utilidad clínica que se puede obtener de un estudio imagenológico en un contexto de bajo riesgo, dichos estudios no aportan mayores beneficios. 
Agradecimientos: Se realiza un agradecimiento al departamento de Anatomía Patológica Clínica Las Condes por la excelente disposición por contribuir al desarrollo de esta investigación.

\section{Referencias}

1. Jemal A, Siegel R, Xu J, Ward E. Cancer statistics, 2010. CA Cancer J Clin 2010; 60: 277-300.

2. Guía Clínica 2010 Cáncer de Próstata en personas de 15 años y más, Ministerio de Salud de Chile. Disponible en: http://www.supersalud.gob.cl/difusion/572/articles-650_guia_clinica.pdf. [Consultado el 12 de junio de 2016].

3. DEIS (Departamento de estadísticas e información de salud). Tasa de Tumores Malignos según Sexo, Chile 1997-2013. Disponible en: http://www.deis.cl/ series-y-graficos-de-mortalidad [Consultado el 12 de junio de 2016].

4. Besa P, Rosso R, Bustos M, Borghero Y, Trucco C, Mac-Namara M. Tratamiento del cáncer de próstata con radioterapia por modulación de intensidad, primera experiencia en Chile. Rev Med Chile 2011; 139 (11): 1451-7.

5. Mottet N, Bellmunt J, Briers E, van den Bergh R, Bolla M, van Casteren N, et al. Guidelines on Prostate Cancer. European Association of Urology 2015. Disponible: http://uroweb.org/wp-content/uploads/09-Prostate-Cancer_LR.pdf. [Consultado el 12 de junio de 2016].

6. Greene KL, Albertsen PC, Babaian RJ, Carter H, Gann $\mathrm{P}$, Han M, et al. Prostate specific antigen best practice statement: 2009 update. J Urol 2009; 182: 2232-41.

7. Mohler J, Armstrong A, Bahnson RR, D'Amico A, Davis B, Eastham J, et al. NCCN Clinical Practice Guidelines in Oncology: Prostate cancer. Version 3. 2016. Disponible: https://www.nccn.org/professionals/physician_gls/ pdf/prostate.pdf [Consultado el 12 de junio de 2016].

8. Gleave $\mathrm{M}$, Coupland D, Drachenberg D, Cohen L, Kwong S, Goldenberg SL, et al. Ability of serum prostate-specific antigen levels to predict normal bone scans in patients with newly diagnosed prostate cancer. Urology 1996; 47 (5): 708-12.

9. Narayan P, Fournier G, Gajendran V, Leidich R, Lo $\mathrm{R}$, Wolf JS Jr, et al. Utility of preoperative serum prostate-specific antigen concentration and biopsy Gleason score in predicting risk of pelvic lymph node metastases in prostate cancer. Urology 1994; 44 (4): 519-24.

10. Briganti A, Passoni N, Ferrari M, Capitanio U, Suardi $\mathrm{N}$, Gallina A, et al. When to Perform Bone Scan in Patients with Newly Diagnosed Prostate Cancer: External
Validation of the Currently Available. Guidelines and Proposal of a Novel Risk Stratification Tool. Eur Urol 2010; 57 (4): 551-8.

11. TNM Classification of Malignant Tumors, $7^{\text {th }}$ edition, 2013. Union for International Cancer Control. Disponible:.www.iucc.org

12. Eifler JB, Feng Z, Lin BM, Partin MT, Humphreys EB, Han $M$, et al. An updated prostate cancer staging nomogram (Partin tables) based on cases from 2006 to 2011. BJU Int 2013; 111 (1): 22-9.

13. Partin AW, Mangold LA, Lamm DM, Walsh PC, Epstein JI, Pearson JD. Contemporary update of the prostate cancer staging nomograms (Partin tables) for the new millennium. Urology 2001; 58 (6): 843-8.

14. Moslehi M, Cheki M, Salehi-Marzijarani M, Amuchastegui T, Gholamrezanezhadet A. Predictors of bone metastasis in pre-treatment staging of asymptomatic treatment-naïve patients with prostate cancer. Rev Esp Med Nucl Imagen Mol 2013; 32 (5): 286-9.

15. Lee N, Fawaaz R, Olsson CA, Benson MC, Petrylak DP, Schiff PB, et al . Which patients with newly diagnosed prostate cancer need a radionuclide bone scan? An analysis based on 631 patients. Int J Radiation Oncology Biol Phys 2000; 48 (5): 1443-6.

16. D'Amico AV, Whittington R, Malkowicz SB, Schultz D, Blank K, Broderick GA, et al. Biochemical outcome after radical prostatectomy, external beam radiation therapy, or interstitial radiation therapy for clinically localized prostate cancer. JAMA 1998; 280 (11): 96974.

17. Levran Z, González JA, Diokno AC, Jafri SZ, Steinert BW. Are pelvic computed tomography, bone scan and pelvic lymphadenectomy necessary in the staging of prostatic cancer? Br J Urol 1995; 75: 778-81.

18. Lavery HJ, Brajtbord JS, Levinson AW, Nabizada-Pace F, Pollard ME, Samadi DB. Unnecessary imaging for the staging of low-risk prostate cancer is common. Urology 2011; 77 (2): 274-8

19. Kindrick AV, Grossfeld GD, Stier DM, Flanders SC, Henning JM, Carroll PR. Use of imaging tests for staging newly diagnosed prostate cancer: trends from the CaPSURE database. J Urol 998; 160: 2102-6.

20. Cooperberg MR, Lubeck DP, Grossfeld GD, Mehta SS, Carroll PR. Contemporary trends in imaging test utilization for prostate cancer staging: data from the cáncer of the prostate strategic urologic research endeavor. J Urol 2002; 168: 491-5.

21. Lu-Yao GL, Friedman M, Yao SL. Use of radical prostatectomy among Medicare beneficiaries before and after the introduction of prostate specific antigen testing. J Urol 1997; 157 (6): 2219. 